Published in final edited form as:

Pharmacoepidemiol Drug Saf. 2018 May ; 27(5): 479-486. doi:10.1002/pds.4291.

\title{
Characterization of Opioid Use in Sickle Cell Disease
}

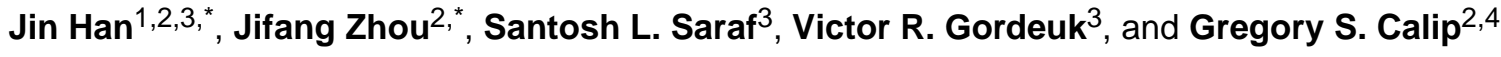 \\ ${ }^{1}$ Department of Pharmacy Practice, College of Pharmacy, University of Illinois at Chicago, \\ Chicago, IL \\ ${ }^{2}$ Center for Pharmacoepidemiology and Pharmacoeconomic Research, University of Illinois at \\ Chicago, Chicago, IL \\ ${ }^{3}$ Comprehensive Sickle Cell Center, Section of Hematology/Oncology, Department of Medicine, \\ University of Illinois at Chicago, Chicago, IL \\ ${ }^{4}$ Division of Public Health Sciences, Epidemiology Program, Fred Hutchinson Cancer Research \\ Center, Seattle, WA, USA
}

\begin{abstract}
Purpose-Opioid analgesics are commonly used to treat vaso-occlusive pain episodes in sickle cell disease (SCD), but comprehensive evidence characterizing opioid use in this patient population is limited. Our objective was to characterize opioid use patterns among SCD patients using a large nationwide database.
\end{abstract}

\begin{abstract}
Methods-A large, U.S. medical claims database was utilized to identify a cohort of 3,882 SCD patients, and characteristics of opioid use were analyzed. Clinical variables including age, gender, medication use, healthcare utilization, and medical history were evaluated for correlations with opioid use.
\end{abstract}

Results-Forty percent of patients took opioid medications during a 12-month span, and the prevalence of any opioid use was highest for 20 to 29 year old patients (58\%). The median daily opioid dose was $1.85 \mathrm{mg}$ (interquartile range: $0.62-10.68 \mathrm{mg}$ ) oral morphine equivalents (OME). While most opioid users took between 0mg and 5mg OME daily, 3\% of pediatric patients and $23 \%$ of adult patients used more than 30mg OME daily. High-dose opioid use was associated with older age, hydroxyurea therapy, nonsteroidal anti-inflammatory drug (NSAID) use, and frequent inpatient hospitalizations. In multivariable-adjusted analyses, patients with vaso-occlusive complications such as pain crisis ( $\mathrm{OR}=3.8,95 \%$ CI 2.7-5.3) and avascular necrosis (AVN) $(\mathrm{OR}=3.7,95 \%$ CI 2.7-5.1) were associated with high-dose opioid use.

Conclusions-Our study showed that only $40 \%$ SCD patients were on opioid analgesics during a 12-month span. However, a non-trivial number of patients used a much higher dose of opioids

\footnotetext{
Address for correspondence: Gregory S. Calip, Center for Pharmacoepidemiology and Pharmacoeconomic Research, University of Illinois at Chicago, 833 South Wood Street MC 871, Chicago, IL 60612, USA.; gcalip@uic.edu.

These authors contributed equally.

Conflict of Interest: The authors declare no conflicts of interest

Prior Presentation:

Results from this study were presented, in part, at the International Society for Pharmacoeconomics and Outcomes Research 22nd Annual International Meeting - May 20-24, 2017, Boston, Massachusetts, USA.
} 
despite a relatively low average daily opioid dose among SCD patients, particularly with vasoocclusive complications.

\section{Introduction}

Sickle cell disease (SCD) is a hemoglobin disorder characterized by homozygous or compound heterozygous mutations in the beta-globin genes. ${ }^{1}$ It affects approximately 100,000 individuals in the U.S. and 25 million people worldwide. ${ }^{2,3}$ Vaso-occlusive crisis (VOC) is one of the signature manifestations in SCD and leads to frequent emergency department (ED) visits and hospital admissions, resulting in an estimated \$2.4 billion in U.S. healthcare costs annually. ${ }^{4}$ Opioid analgesics are a mainstay of pharmacotherapy in treating pain episodes in $\mathrm{SCD},{ }^{1}$ although doses and types of opiate medications vary greatly among SCD patients. ${ }^{5}$ The evidence on the patterns of opioid use in this patient population is rather limited, and there is still controversy on the dose of opioids that SCD patients use in their daily life. ${ }^{6,7}$ Many believe that patients with SCD tend to use more opioids than many other chronic pain conditions, ${ }^{7}$ whereas some studies showed a relatively low opioid dose used in this patient population in their daily life. ${ }^{5,8,9}$ But most of the previous studies were based on single-institutions with relatively small sample sizes or based on a self-reported questionnaire or diary, $5,8,9$ which may limit the generalizability of the results. There are no studies investigating the use of opioids in both pediatric and adult patients. Therefore, a comprehensive evaluation of opioid use patterns among SCD patients using a national database is warranted.

We found that the opioid doses used by the adult SCD patients were approximately $6 \mathrm{mg}$ oral morphine equivalents (OME) in a single-institution cohort, and the doses of opioids were correlated with vaso-occlusive complications such as pain crisis and avascular necrosis (AVN). ${ }^{5}$ In this study, we utilized a large nationwide medical/pharmacy claim database to confirm the previous findings and test our hypothesis that SCD patients might use relatively low doses of opioids in their daily life, and a subset of patients with certain clinical characteristics might have much higher dosing requirements. The findings presented here will help to better understand the characteristics of SCD patients requiring higher doses of opioids in real-world settings and optimize pain management and intervention strategies.

\section{Methods}

\section{Data source}

Data for this analysis were collected from the Truven Health Analytics MarketScan ${ }^{\circledR}$

Commercial Claims and Encounters database and the Medicare Supplemental and Coordination of Benefits database between 2009 and 2014. This administrative database contains information on patient demographics, enrollment history, claims for pharmacy dispensing records, and inpatient and outpatient services. All data were de-identified and complied with Health Insurance Portability and Accountability Act (HIPAA) privacy rules. This study was determined to be exempt by the Institutional Review Board of the University of Illinois at Chicago. 


\section{Patient population}

We conducted a retrospective cohort study of patients who were identified as having SCD using a validated SCD-identifying algorithm. ${ }^{10}$ In brief, if at least one claim with an International Classification of Disease, Ninth Revision, Clinical Modification (ICD-9-CM) diagnostic code for SCD from an acute care hospital encounter (see Supplemental table 1 for codes) or two separate claims with ICD-9-CM SCD diagnostic codes that were at least 30 days apart were present, then the individual was preliminarily defined as having SCD. An additional SCD-identifying algorithm (RuSH) was also applied to the cohort to further select probable SCD cases (Supplemental table 2). ${ }^{11}$ Patients were required to have 12 months of baseline continuous enrollment in their respective health plan with 12 additional months of continuous enrollment during the follow-up period. Patients were excluded if they were diagnosed with sickle cell trait, had any type of cancer, or received a hematopoietic stem cell or solid organ transplantation. Selection of patients for this analysis is summarized in a CONSORT diagram (Figure 1).

ICD-9-CM codes (Supplemental table 3) were used to identify inpatient and outpatient encounters, medical conditions and procedures. The study period consisted of a baseline (months 0-12) and observation period (months 13-24). Information on characteristics including age, gender, concomitant nonsteroidal anti-inflammatory drug (NSAID) and hydroxyurea use, history of medical conditions, and history of healthcare utilization (hospital admission and ED visit) were collected at the baseline period (months 0-12); information on opioid use was collected during the observation period (months 13-24). The clinical information collected from the baseline period was utilized to examine the correlation with opioid use in the observation period.

\section{Opioid medication dose conversion and group categorization}

Information on opioid medication doses were determined from pharmacy dispensing records and converted to oral morphine equivalents (OME) based on the following ratio: $1 \mathrm{mg}$ codeine $=0.13 \mathrm{mg}$ OME, $1 \mathrm{mg}$ hydrocodone $=1 \mathrm{mg}$ OME, $1 \mathrm{mg}$ hydromorphone $=5 \mathrm{mg}$ OME, $1 \mathrm{mg}$ methadone $=4.7 \mathrm{mg}$ OME, $1 \mathrm{mg}$ tramadol $=0.2 \mathrm{mg}$ OME, $1 \mathrm{mg}$ oxycodone $=$ $1.5 \mathrm{mg}$ OME, $1 \mathrm{mg}$ tapentadol $=0.4 \mathrm{mg}$ OME, $1 \mathrm{mg}$ meperidine $=0.1 \mathrm{mg}$ OME, $1 \mathrm{mg}$ oxymorphone $=3 \mathrm{mg}$ OME, $1 \mathrm{mg}$ pentazocine $=0.37 \mathrm{mg}$ OME, $1 \mathrm{mg}$ propoxyphene $=0.08$ $\mathrm{mg}$ OME, $1 \mathrm{mcg} / \mathrm{hr}$ transdermal fentanyl = $3 \mathrm{mg}$ OME$/ \mathrm{day}, 1 \mathrm{mcg} / \mathrm{hr}$ transdermal buprenorphine patch $=2.5 \mathrm{mg}$ OME/day. Approximately $10 \%$ of the opioid prescriptions lacked detailed dosage information and were excluded from the OME calculation. Opioid users were categorized into non/low-dose users ( $24.5 \mathrm{mg}$ OME daily in the adult population with $\mathrm{SCD}$ ) and high-dose users ( $>24.5 \mathrm{mg}$ OME daily in the adult population with SCD). The cutoff of $24.5 \mathrm{mg}$ OME was based on the $75^{\text {th }}$ percentile of the average daily oral opioid dose.

\section{Statistical analysis}

In the univariate analysis, the characteristics of SCD patients including age, gender, medication use, healthcare utilization, and medical history were compared between non/lowdose users and high-dose users using Chi-square test or Fisher's exact test for cells with expected frequencies $<5$ for categorical variables; continuous variables were compared 
using the two-sample Student's t-test or Kruskal-Wallis test. In the multivariate analysis, a stepwise multiple logistic regression was performed. The opioid dose categories (non/lowdose users and high-dose users) were dependent variables, and age, gender, medication use, healthcare utilization, and medical history were independent variables. Covariates with $p$ > 0.20 were eliminated from the model in a stepwise manner. Adjusted odds ratios (OR) and 95\% confidence intervals (CI) for likelihood of being a non/low-dose users or high-dose opioid users were calculated. All analyses were two-sided and conducted using SAS 9.3 (SAS Institute Inc. Cary, NC, USA) and open-source software R 3.3.1 including the NNET package (https://CRAN.R-project.org).

\section{Results}

A total of 3,882 patients with a median age of 17 years (interquartile range [IQR]: 9-36 years) were included in our analysis. Forty-five percent of patients were male. Opioid medications were used by $39.9 \%$ of patients, $31.8 \%$ taking only immediate-release opioids, $0.4 \%$ taking only extended-release opioids, and $7.8 \%$ using both immediate-release and extended-release formulations (Table 1). The prevalence of opioid use was $8.5 \%$ in the $0-9$ years age group, increased significantly to $46.3 \%$ in the $10-19$ years age group ( $\mathrm{p}<0.0001$ ), and peaked at $58.3 \%$ in the $20-29$ years age group.

Doses of opioid medications converted to oral morphine equivalent (OME) are reported in Table 2. The median daily dose among opioid users was $1.85 \mathrm{mg}$ OME (IQR 0.62-10.68 $\mathrm{mg}$ ). The dose of opioids was $0.54 \mathrm{mg}$ OME per day in the age groups of 0-9 years old, and significantly higher at $1.07 \mathrm{mg}(\mathrm{p}=0.0002)$ in the $10-19$ years old. The age group of $50+$ years old had the highest opioid dose (6.03 $\mathrm{mg}$ OME per day). In the pediatric patients (017 years old) on opioid medications, the vast majority (87\%) used 0-5 mg OME per day, and only $3 \%$ used more than $30 \mathrm{mg}$ OME daily (Figure 2). In contrast, $55 \%$ of the adult patients (18+ years old) used 0-5 mg OME per day, and 23\% took more than $30 \mathrm{mg}$ OME daily. The daily dose of $30 \mathrm{mg}$ OME daily was used as the highest cutoff here since it was a commonly used dosage form for morphine. Hydrocodone- and oxycodone-containing medications were among the most commonly used opioids in both pediatric and adult patients (Figure 3), each accounting for approximately $30 \%$ of the total number of opioid prescriptions. Codeine-containing medications were more frequently used in the pediatric population (27\%) compared to adult population (4\%). The usage of stronger opioid medications such as morphine, hydromorphone, methadone, and fentanyl was higher in the adult patients (27\%) compared to the pediatric patients (16\%).

Descriptive and clinical characteristics of adult patients (18 years and older) by non/lowdose use and high-dose average daily opioid use are reported in Supplementary Table 4. Older age, hydroxyurea use, and NSAID use were more common among high-dose opioids users. Hospitalizations and ED visits were also more common among high-dose opioid users. High-dose opioid users experienced a greater incidence of VOCs $(\mathrm{p}<0.0001)$, acute chest syndrome $($ ACS $)(p=0.0211), A V N(p<0.0001)$, and chronic heart failure $(p=$ 0.0027). 
In the multivariable logistic regression models, patients that were older, used hydroxyurea (OR 3.9, 95\% CI 2.8-5.5), and used NSAIDs (OR 2.0, 95\% CI 1.4-2.7) had significantly higher odds of high-dose opioid use (Table 3). Greater frequency of hospitalizations (2+ admissions annually) was significantly associated with high-dose (OR 4.7, 95\% CI 3.2-6.9) opioid use. In a separate multivariable regression model (Table 4), the medical condition associated with high-dose opioid users were VOC (OR 3.8, 95\% CI 2.7-5.3) and AVN (OR $3.7,95 \%$ CI 2.7-5.1), suggesting that opioid use may be associated with vaso-occlusive complications.

\section{Discussion}

Our findings showed that $39.9 \%$ of patients used opioid medications during a 12-month period, and the median dose among opioid users was $1.85 \mathrm{mg}$ OME per day. We also found wide variations of opioid use in this patient population. While most patients (87\% in pediatric patients and 55\% in adult patients) used less than $5 \mathrm{mg}$ OME daily, a non-trivial number of patients used more than $30 \mathrm{mg}$ OME daily (3\% and $23 \%$ in pediatric and adult patients, respectively). High-dose opioid use was associated with older age, use of hydroxyurea or NSAIDs, and these patients tended to have a greater frequency of hospital admissions. History of AVN and occurrence of VOCs were also associated with high-dose opioid use.

Opioid analgesic medications are the main treatment options for acute and chronic pain caused by $\mathrm{SCD},{ }^{12}$ although the literature on the patterns of opioid use in this patient population is rather limited. ${ }^{5,8,13,14}$ Most studies were based on a self-reported questionnaire or diary with relatively small numbers of patients, which may be subject to recall or selection bias. ${ }^{15}$ In this study, an administrative medical and pharmacy claims database was utilized to identify a large number of subjects with SCD and pharmacy dispensing records were extracted to evaluate patterns of opioid use. There is a misconception that patients with SCD tend to use more opioids than other chronic pain syndromes. ${ }^{7}$ Our results here showed that the majority of patients with SCD (60.1\%) did not use opioids on a daily basis, either because some SCD patients had pain but did not require opioids or because not all SCD patients experience daily pain. Previous studies showed that SCD patients have self-reported pain in $17-55 \%$ of the observed days. ${ }^{16-18}$ The calculated average daily opioid dose in this study was relatively low (less than $2 \mathrm{mg}$ OME) among opioid users. Although patients in this cohort were younger (median age 17 years old), the daily opioid dose was similar at 4 $\mathrm{mg}$ OME in the adult patients (18+ years). Another consideration is that the subjects included in our analysis were all enrollees of private or employer-based insurance plans and they tend to have fewer complications and lower healthcare utilization compared to patients enrolled on publicly-insured health plans. ${ }^{19,20}$ Another study on a cohort of mainly publiclyinsured adult patients with SCD indicated that the median dose of daily OME was $6 \mathrm{mg},{ }^{5}$ which is similar to our observation in this study and lower than the dose used to treat other pain syndromes, such as chronic non-cancer pain. ${ }^{21,22}$

On the high end of the opioid use spectrum, our results characterized a group of SCD patients (3\% in 0-17 year old group and $23 \%$ in $18+$ year old group) requiring more than 30 mg OME daily opioid use, and this is consistent with previous reports showing a cluster of 
high healthcare utilizers among SCD patients. ${ }^{23,24}$ When treating chronic and acute pain in these patients, high doses of opioids are probably warranted to achieve adequate pain control and legitimate opioid use should not be denied due to the current national opioid phobia. Although opioid-related deaths or severe adverse events cannot be determined in this study, it has been shown that using high-doses of opioids to treat VOC in SCD patients is a relatively safe approach. ${ }^{25}$ We identified several associations with opioid use in this study population that support the use of higher doses in patients with worse disease. Hydroxyurea is indicated for SCD patients with recurrent moderate-to-severe painful crises, ${ }^{26}$ and its use was indeed associated with high-dose opioid use. VOCs are usually treated with a multimodal approach, which was manifested by the association between opioid use and NSAID use. The results here showed that compared to non/low-dose users, high-dose opioid users were associated with frequent hospital admissions which emphasizes the importance of setting up a day hospital dedicated to making prompt and adequate pain treatment available to SCD patients. Experience from other hospitals and our own institution have shown that a day hospital can be an effective approach to manage this cluster of high healthcare utilizers by improving timeliness of treatment, decreasing ED visits, and reducing hospital admission rates. ${ }^{23,27}$

Vaso-occlusion and hemolysis each have a distinctive pathophysiology contributing to some sub-phenotypes of SCD. ${ }^{28}$ Pain crises and AVN are believed to be related to the vasoocclusion mechanism, whereas hemolysis is associated with complications such as pulmonary hypertension, stroke, and priapism. ${ }^{28}$ Our observation that high-dose opioid use was associated with vaso-occlusive complications (VOCs and AVN), but not hemolytic complications (pulmonary hypertension, stroke, and priapism) suggests that opioid use is related more with vaso-occlusion rather than hemolysis-related mechanism. AVN is a relatively prevalent complication among the $\mathrm{SCD}$ patients ${ }^{29}$, resulting in severe chronic pain and disability, and it negatively affects health-related quality of life ${ }^{30}$. Early interventions, such as referring for orthopedic surgery procedures and/or physical therapy, may be warranted to reduce the opioid requirement in SCD patients with AVN. High-dose opioid use was associated with ACS and chronic heart failure in univariate but not in multivariate analyses (supplemental table 4). Exposure to high-dose morphine and its active metabolite, morphine-6-glucuronide, has been suggested to increase the risk of ACS in SCD patients, ${ }^{31}$ which is consistent with our observation. Opioid use has also been associated with adverse respiratory outcomes, ${ }^{32,33}$ but not heart failure in other patient populations. ${ }^{34,35}$

Both the prevalence and dose of opioids increased significantly from the $0-9$ age group to the 10-19 year age group, implying that the vaso-occlusive pain increases during the second decade of life in the SCD patients. The prevalence of opioid use slowly declined with increasing age after reaching the peak at the 20-29 years group, especially in the age group of 50+ years old (Table 1). The reduced opioid use prevalence observed in the older age groups might result from a survival effect: patients with no opioid use may have less severe disease and hence lower mortality rates. This is consistent with findings that the pain severity rate in patients with SCD declines after 30 years of age, and patients with frequent VOCs have higher mortality risk. ${ }^{36}$ On the other hand, the median dose among opioid users increased between ages 0 and 29 years old, plateaued in the third and fourth decades, and then reached the highest levels in the 50+ year age group (Table 2). This may be due to the 
higher likelihood of developing AVN, which increases with age and was associated with greater opioid use in this study. ${ }^{37}$ Additionally, patients with high quality medical care may have improved access to opioid treatment to manage VOCs, and they in turn may have a longer life expectancy. As patients with SCD live a longer life into their 50-60s in average, sometimes even 80 s with improvement in medical care, ${ }^{38-41}$ it is important to investigate alternative approaches for pain management to complement the pain treatment regimen. Non-opioid based treatments including both pharmacological and non-pharmacological approaches are particularly important for the cluster of SCD patients who fail to respond to the opioid therapies.

\section{Study limitations}

Our study is not without limitations. The results are based on analysis of pharmacy dispensing records, which may overestimate opioid use since not all dispensed medications are consumed by the patients or taken as directed. Patients were included in this analysis if they had at least one diagnostic code for SCD in an acute care hospital encounter or two separate diagnostic codes 30 days apart, which may have excluded some patients with less severe disease that utilize healthcare infrequently. This study is based on privately-insured subjects, which may be different from publicly-insured patients, but it still provided important information about characteristics of relatively healthy SCD patients as previous studies demonstrated. ${ }^{19,42}$ The difference in medication utilization between privately- and publicly-insured patients should be addressed in further studies. Despite these potential limitations, our analysis identified several associations with opioid use, and it is the first study characterizing patterns of opioid use in both pediatric and adult SCD patients using a large nationwide database.

\section{Conclusion}

In summary, our results showed that on the whole, patients with SCD used a relatively low dose of opioids in their daily life, while a subset of patients had much higher opioid dose requirements. The high-dose opioid users were associated with vaso-occlusive complications, such as VOC and AVN, and tended to seek for healthcare utilization more frequently. They may contribute to the vast majority of healthcare costs associated with $\mathrm{SCD}$; therefore, it is important to optimize the pain management in this subset of patients to reduce the healthcare costs. Early interventions for potentially modifiable complications such as AVN may help decrease high-dose opioid use in this patient population.

\section{Supplementary Material}

Refer to Web version on PubMed Central for supplementary material.

\section{Acknowledgments}

Dr. Zhou was supported by the University of Illinois at Chicago-AbbVie Fellowship in Health Economics and Outcomes Research. Dr. Calip was supported by the National Center for Advancing Translational Sciences, National Institutes of Health, through Grant UL1TR002003. The content is solely the responsibility of the authors and does not necessarily represent the official views of the National Institutes of Health. 


\section{References}

1. Steinberg MH. Management of sickle cell disease. N Engl J Med. 1999; 340:1021-30. [PubMed: 10099145]

2. Hassell KL. Population estimates of sickle cell disease in the US. Am J Prev Med. 2010; 38:S51221. [PubMed: 20331952]

3. Modell B, Darlison M. Global epidemiology of haemoglobin disorders and derived service indicators. Bull World Health Organ. 2008; 86:480-7. [PubMed: 18568278]

4. Lanzkron S, Carroll CP, Haywood C Jr. The burden of emergency department use for sickle-cell disease: an analysis of the national emergency department sample database. Am J Hematol. 2010; 85:797-9. [PubMed: 20730795]

5. Han J, Saraf SL, Zhang X, et al. Patterns of opioid use in sickle cell disease. Am J Hematol. 2016; 91:1102-6. [PubMed: 27466799]

6. Han J, Saraf SL, Machado RF, Gordeuk VR. Reply to Ruan X et al: “A comment on pattern of opioid use in sickle cell disease". Am J Hematol. 2017

7. Ruan X, Wu H, Wang D. A Comment on Pattern of Opioid Use in Sickle Cell Disease. Am J Hematol. 2017

8. Smith WR, McClish DK, Dahman BA, et al. Daily home opioid use in adults with sickle cell disease: The PiSCES project. J Opioid Manag. 2015; 11:243-53. [PubMed: 25985809]

9. Ballas SK, Bauserman RL, McCarthy WF, Castro OL, Smith WR, Waclawiw MA. Utilization of analgesics in the multicenter study of hydroxyurea in sickle cell anemia: effect of sex, age, and geographical location. Am J Hematol. 2010; 85:613-6. [PubMed: 20568300]

10. Michalik DE, Taylor BW, Panepinto JA. Identification and Validation of a Sickle Cell Disease Cohort Within Electronic Health Records. Acad Pediatr. 2016

11. Paulukonis ST, Harris WT, Coates TD, et al. Population based surveillance in sickle cell disease: methods, findings and implications from the California registry and surveillance system in hemoglobinopathies project (RuSH). Pediatr Blood Cancer. 2014; 61:2271-6. [PubMed: 25176145]

12. Ballas SK, Gupta K, Adams-Graves P. Sickle cell pain: a critical reappraisal. Blood. 2012; 120:3647-56. [PubMed: 22923496]

13. Yoon SL, Black S. Comprehensive, integrative management of pain for patients with sickle-cell disease. J Altern Complement Med. 2006; 12:995-1001. [PubMed: 17212571]

14. Dampier C, Ely E, Brodecki D, O’Neal P. Home management of pain in sickle cell disease: a daily diary study in children and adolescents. J Pediatr Hematol Oncol. 2002; 24:643-7. [PubMed: 12439036]

15. Huber LR, Broel EC, Mitchelides AN, Dmochowski J, Dulin M, Scholes D. Comparison of prospective daily diaries and retrospective recall to measure oral contraceptive adherence. Contraception. 2013; 88:492-7. [PubMed: 23582236]

16. van Tuijn CF, Sins JW, Fijnvandraat K, Biemond BJ. Daily pain in adults with sickle cell disease-a different perspective. Am J Hematol. 2017; 92:179-86. [PubMed: 27880985]

17. Smith WR, Penberthy LT, Bovbjerg VE, et al. Daily assessment of pain in adults with sickle cell disease. Ann Intern Med. 2008; 148:94-101. [PubMed: 18195334]

18. Porter LS, Gil KM, Sedway JA, Ready J, Workman E, Thompson RJ Jr. Pain and stress in sickle cell disease: an analysis of daily pain records. Int J Behav Med. 1998; 5:185-203. [PubMed: 16250701]

19. Mvundura M, Amendah D, Kavanagh PL, Sprinz PG, Grosse SD. Health care utilization and expenditures for privately and publicly insured children with sickle cell disease in the United States. Pediatr Blood Cancer. 2009; 53:642-6. [PubMed: 19492318]

20. Robinson MR, Daniel LC, O’Hara EA, Szabo MM, Barakat LP. Insurance status as a sociodemographic risk factor for functional outcomes and health-related quality of life among youth with sickle cell disease. J Pediatr Hematol Oncol. 2014; 36:51-6. [PubMed: 24136028]

21. Marschall U, L'Hoest H, Radbruch L, Hauser W. Long-term opioid therapy for chronic non-cancer pain in Germany. Eur J Pain. 2016; 20:767-76. [PubMed: 26492066] 
22. Campbell G, Nielsen S, Larance B, et al. Pharmaceutical Opioid Use and Dependence among People Living with Chronic Pain: Associations Observed within the Pain and Opioids in Treatment (POINT) Cohort. Pain Med. 2015; 16:1745-58. [PubMed: 26011277]

23. Koch KL, Karafin MS, Simpson P, Field JJ. Intensive management of high-utilizing adults with sickle cell disease lowers admissions. Am J Hematol. 2015; 90:215-9. [PubMed: 25469750]

24. Carroll CP, Haywood C Jr, Fagan P, Lanzkron S. The course and correlates of high hospital utilization in sickle cell disease: Evidence from a large, urban Medicaid managed care organization. Am J Hematol. 2009; 84:666-70. [PubMed: 19743465]

25. Tanabe P, Martinovich Z, Buckley B, Schmelzer A, Paice JA. Safety of an ED High-Dose Opioid Protocol for Sickle Cell Disease Pain. J Emerg Nurs. 2015; 41:227-35. [PubMed: 25241635]

26. Platt OS. Hydroxyurea for the treatment of sickle cell anemia. N Engl J Med. 2008; 358:1362-9. [PubMed: 18367739]

27. Benjamin LJ, Swinson GI, Nagel RL. Sickle cell anemia day hospital: an approach for the management of uncomplicated painful crises. Blood. 2000; 95:1130-6. [PubMed: 10666181]

28. Kato GJ, Gladwin MT, Steinberg MH. Deconstructing sickle cell disease: reappraisal of the role of hemolysis in the development of clinical subphenotypes. Blood Rev. 2007; 21:37-47. [PubMed: 17084951]

29. Milner PF, Kraus AP, Sebes JI, et al. Sickle cell disease as a cause of osteonecrosis of the femoral head. N Engl J Med. 1991; 325:1476-81. [PubMed: 1944426]

30. Dampier C, Lieff S, LeBeau P, et al. Health-related quality of life in children with sickle cell disease: a report from the Comprehensive Sickle Cell Centers Clinical Trial Consortium. Pediatr Blood Cancer. 2010; 55:485-94. [PubMed: 20658620]

31. Kopecky EA, Jacobson S, Joshi P, Koren G. Systemic exposure to morphine and the risk of acute chest syndrome in sickle cell disease. Clin Pharmacol Ther. 2004; 75:140-6. [PubMed: 15001964]

32. Dublin S, Walker RL, Jackson ML, et al. Use of opioids or benzodiazepines and risk of pneumonia in older adults: a population-based case-control study. J Am Geriatr Soc. 2011; 59:1899-907. [PubMed: 22091503]

33. Vozoris NT, Wang X, Fischer HD, et al. Incident opioid drug use and adverse respiratory outcomes among older adults with COPD. Eur Respir J. 2016; 48:683-93. [PubMed: 27418553]

34. Gupta T, Mujib M, Agarwal P, et al. Association Between Opioid Abuse/Dependence and Outcomes in Hospitalized Heart Failure Patients. Am J Ther. 2016; 23:e350-6. [PubMed: 25611362]

35. Dawson NL, Roth V, Hodge DO, Vargas ER, Burton MC. Opioid Use in Patients with Congestive Heart Failure. Pain Med. 2017

36. Platt OS, Thorington BD, Brambilla DJ, et al. Pain in sickle cell disease. Rates and risk factors. N Engl J Med. 1991; 325:11-6. [PubMed: 1710777]

37. Mukisi-Mukaza M, Elbaz A, Samuel-Leborgne Y, et al. Prevalence, clinical features, and risk factors of osteonecrosis of the femoral head among adults with sickle cell disease. Orthopedics. 2000; 23:357-63. [PubMed: 10791585]

38. Platt OS, Brambilla DJ, Rosse WF, et al. Mortality in sickle cell disease. Life expectancy and risk factors for early death. N Engl J Med. 1994; 330:1639-44. [PubMed: 7993409]

39. Wierenga KJ, Hambleton IR, Lewis NA. Survival estimates for patients with homozygous sicklecell disease in Jamaica: a clinic-based population study. Lancet. 2001; 357:680-3. [PubMed: 11247552]

40. Quinn CT, Rogers ZR, McCavit TL, Buchanan GR. Improved survival of children and adolescents with sickle cell disease. Blood. 2010; 115:3447-52. [PubMed: 20194891]

41. Ballas SK, Pulte ED, Lobo C, Riddick-Burden G. Case series of octogenarians with sickle cell disease. Blood. 2016

42. Stettler N, McKiernan CM, Melin CQ, Adejoro OO, Walczak NB. Proportion of adults with sickle cell anemia and pain crises receiving hydroxyurea. JAMA. 2015; 313:1671-2. [PubMed: 25919532] 


\section{Key Points}

- Only $40 \%$ of SCD patients were on opioid analgesics during a 12-month span;

- Majority of the patients with SCD used relatively low doses of opioids in their daily life, whereas a cluster of patients had much higher dosing requirements;

- High-dose opioid users tended to utilize healthcare more frequently;

- Vaso-occlusive complications such as VOC and AVN were associated with high-dose opioid use. 


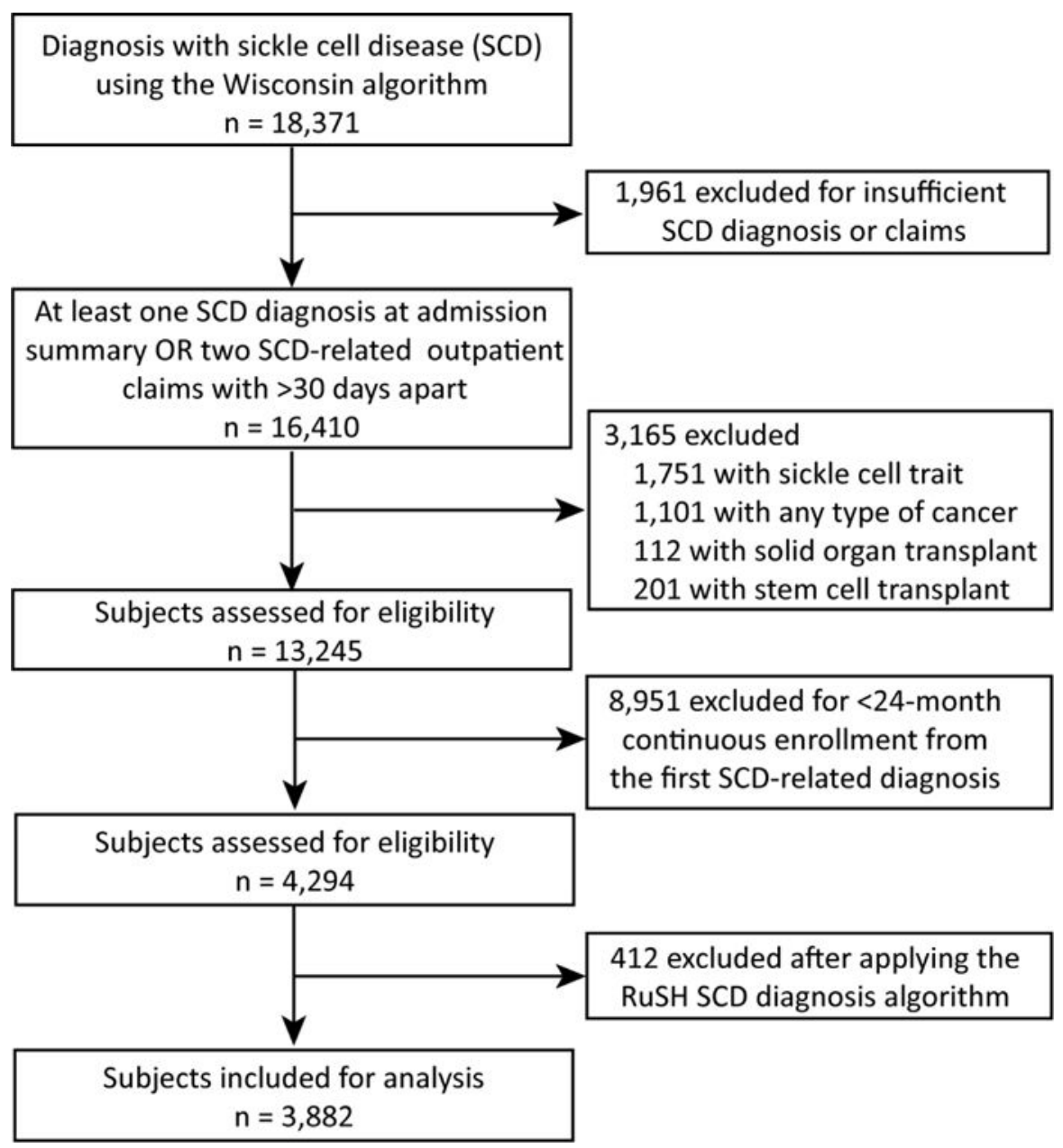

Figure 1.

Flow-chart of patient inclusion in the study. 


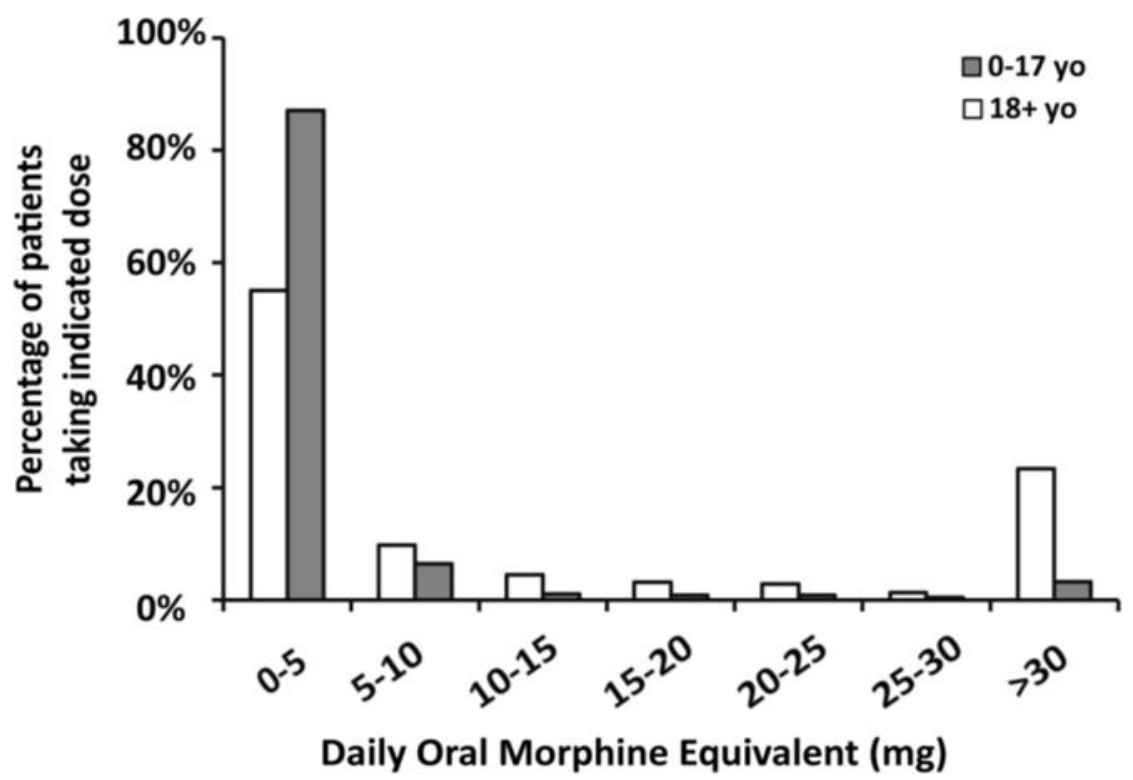

Figure 2.

Frequency of opioid use in the SCD patients. 

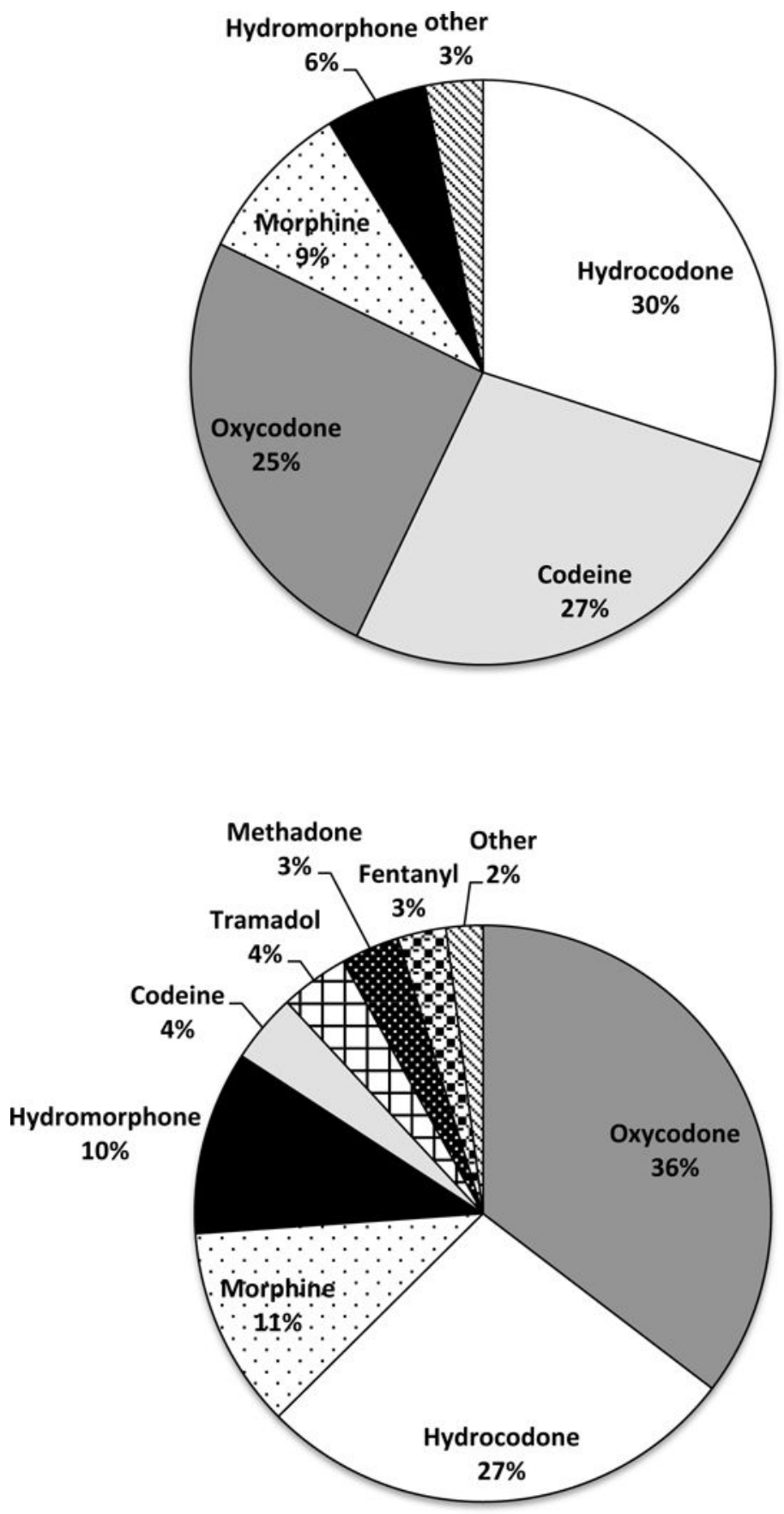

Figure 3.

Proportion of opioid prescriptions among SCD patients. 


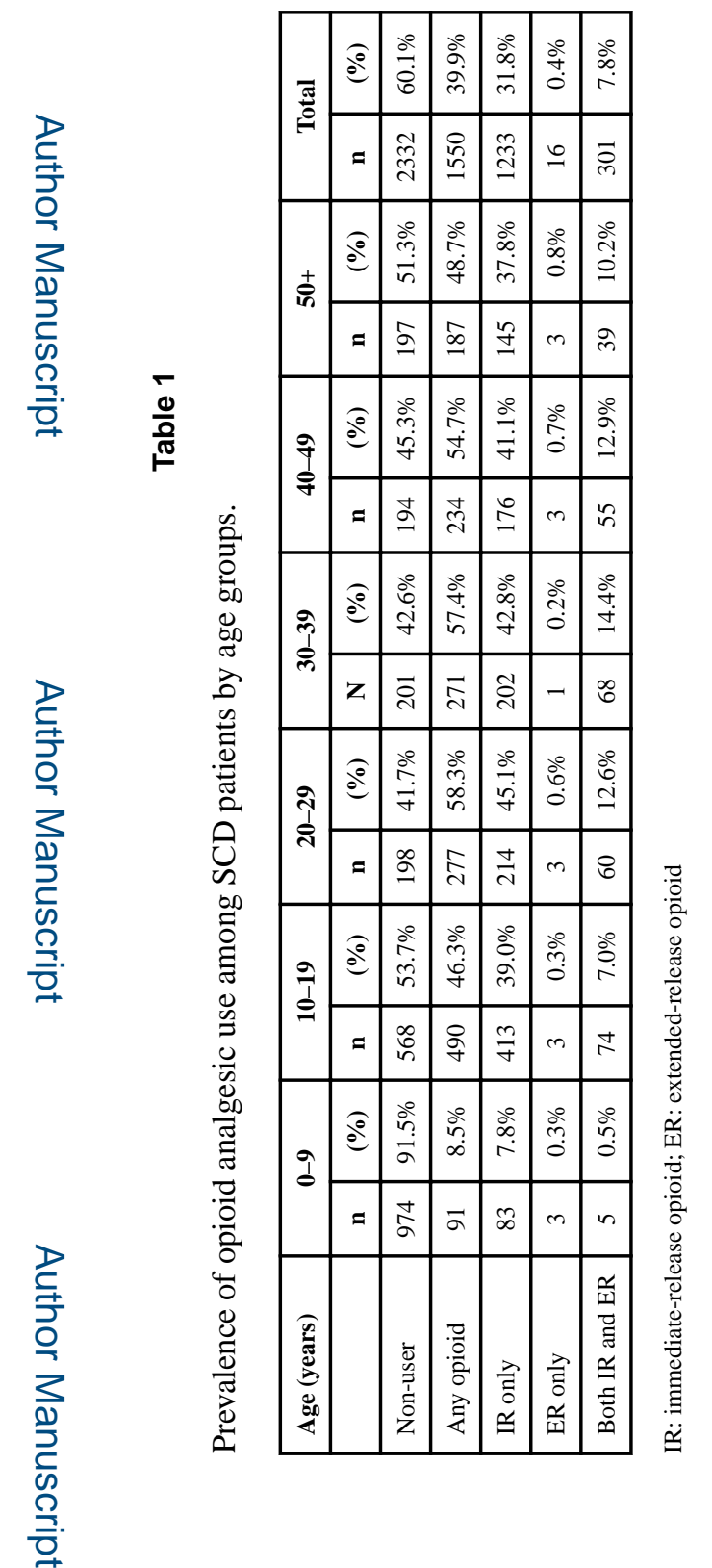

Pharmacoepidemiol Drug Saf. Author manuscript; available in PMC 2019 May 01. 


\section{Table 3}

Clinical characteristics independently associated with high-dose opioid use.

\begin{tabular}{|l|c|c|c|}
\hline Correlate & Odds ratio & 95\% CI & p-value \\
\hline Age (years) & & & \\
\hline $18-29$ (reference) & 1.0 & Reference & NA \\
\hline $30-39$ & 1.9 & $1.3-2.9$ & 0.0025 \\
\hline $40-49$ & 2.6 & $1.7-3.9$ & $<0.0001$ \\
\hline $50+$ & 2.7 & $1.7-4.2$ & $<0.0001$ \\
\hline Gender (M vs. F) & 1.4 & $1.0-1.9$ & 0.03780 \\
\hline Hydroxyurea user & 3.9 & $2.8-5.5$ & $<0.0001$ \\
\hline NSAID user & 2.0 & $1.4-2.7$ & $<0.0001$ \\
\hline Inpatient hospitalizations & & & \\
\hline 0 admissions (reference) & 1.0 & Reference & NA \\
\hline 1 admissions & 1.7 & $1.1-2.6$ & 0.0132 \\
\hline 2+ admissions & 4.7 & $3.2-6.9$ & $<0.0001$ \\
\hline Emergency department visits & & & \\
\hline 0 ED visits (reference) & 1.0 & Reference & NA \\
\hline $1-2$ ED visits & 1.2 & $0.8-1.7$ & 0.3395 \\
\hline 3+ ED visits & 1.6 & $1.1-2.4$ & 0.0258 \\
\hline Transfusion history & 1.4 & $0.9-2.1$ & 0.1004 \\
\hline
\end{tabular}

Multivariate logistic regression was performed based on opioid dose category. 


\section{Table 4}

Medical conditions independently associated with high-dose opioid use.

\begin{tabular}{|l|c|c|c|}
\hline Correlate & Odds ratio & $\mathbf{9 5 \%}$ CI & p-value \\
\hline Acute renal injury & 1.7 & $1.0-3.0$ & 0.0598 \\
\hline Avascular necrosis & 3.7 & $2.7-5.1$ & $<0.0001$ \\
\hline Iron overload & 1.8 & $1.1-2.8$ & 0.0111 \\
\hline Vaso-occlusive crisis & 3.8 & $2.7-5.3$ & $<0.0001$ \\
\hline
\end{tabular}

A stepwise multiple logistic regression was performed based on opioid dose category (non/low-dose users vs high-dose users). The covariates originally placed into the analysis were chronic heart failure, chronic renal disease, stroke, pulmonary hypertension, avascular necrosis, priapism, iron overload, vaso-occlusive crisis, acute chest syndrome, and acute renal disease. Acute renal injury, avascular necrosis, iron overload, and vasoocclusive crisis remained in the regression model after covariates with $p>0.20$ were eliminated from the model. Chronic heart failure, chronic renal disease, stroke, pulmonary hypertension, priapism, and acute chest syndrome eliminated from the model in a stepwise manner. The model was adjusted for age and gender. 